\title{
Observability of Higgs mode in a system without Lorentz invariance
}

\author{
Xinloong Han, ${ }^{1}$ Boyang Liu, ${ }^{2, *}$ and Jiangping $\mathrm{Hu}^{1,3}$ \\ ${ }^{1}$ Institute of Physics, Chinese Academy of Sciences, Beijing 100190, China \\ ${ }^{2}$ Department of Physics and Center of Theoretical and Computational Physics, The University of Hong Kong, Hong Kong, China \\ ${ }^{3}$ Collaborative Innovation Center of Quantum Matter, Beijing 100190, China
}

(Received 5 May 2016; published 8 September 2016)

\begin{abstract}
We study the observability of the Higgs mode in BEC-BCS crossover. The observability of the Higgs mode is investigated by calculating the spectral weight functions of the amplitude fluctuation below the critical transition temperature. At zero temperature, we find that there are two sharp peaks on the spectral function of the amplitude fluctuation attributed to Goldstone and Higgs modes, respectively. As the system goes from the BCS to BoseEinstein condensate (BEC) side, there is strong enhancement of spectral weight transfer from the Higgs to Goldstone mode. However, even at the unitary regime where the Lorentz invariance is lost, the sharp feature of the Higgs mode still exists. We specifically calculate the finite-temperature spectral function of the amplitude fluctuation at the unitary regime and show that the Higgs mode is observable at the temperature that present experiments can reach.
\end{abstract}

DOI: 10.1103/PhysRevA.94.033608

\section{INTRODUCTION}

A general description of many-body theory shows that after a spontaneous symmetry breaking two kinds of collective modes emerge [1]. One is known as the Goldstone mode and originates from the phase fluctuation of order parameters. The other one is referred to as the "Higgs mode," which is from the amplitude fluctuation of the order parameter. With the triumph of the observation of the Higgs particle in high-energy physics [2,3], the interest in Higgs collective modes in many-body systems is growing. The earliest observation was in the Raman scattering experiment of a superconducting charge-density wave compound $\mathrm{NbSe}_{2}$ in the 1980s [4-6]. Recently, with the development of experimental techniques more evidence of the Higgs mode in many-body systems has appeared. For example, the Higgs mode has been observed in the antiferromagnet $\mathrm{TlCuCl}_{3}$ via neutron scattering [7], and terahertz pump-probe spectroscopy also revealed the signal of the Higgs mode in a superconducting $\mathrm{NbN}$ sample in a nonadiabatic excitation regime [8,9]. In a cold-atom system, the Higgs mode has been observed in bosonic atoms in optical lattices when fine-tuning the parameters to the Lorentz invariance point [10]. Various theoretical studies have also been conducted [11-22].

Usually, the existence of the Higgs mode requires Lorentz invariance. In Ref. [21] one of us manifestly discussed how the Higgs mode evolved as the system was tuned away from the Lorentz-invariance point. Basically, as Lorentz invariance is gradually lost, the spectral weight transfer from the Higgs mode to the Goldstone mode is enhanced; meanwhile, the Higgs mode becomes more strongly coupled to the quasiparticle excitations and other collective modes and finally gets overdamped. Hence, close to the unitary regime, the Higgs mode is nonobservable. However, in Ref. [21] the whole derivation is based on the time-dependent GinzburgLandau theory. The validity is constrained in the vicinity of the transition temperature, where the damping effect is strong compared with the case of lower temperature. It is

\footnotetext{
*boyangleo@gmail.com
}

interesting to investigate the observability of the Higgs mode using a mean-field theory in the case deep below the critical temperature.

In this work we study the Fermion superfluid of dilute Fermi gas with tunable interaction. In our theoretical framework the superfluid order parameter is represented as the mean field plus the phase and amplitude fluctuations. The spectral weight functions of the phase and amplitude fluctuations can be calculated using the functional integral method. First, we discuss the spectral weight transfer of the Higgs mode and Goldstone mode at zero temperature and show that the signal of the Higgs mode is still clear in the unitary regime even though the Lorentz invariance is lost here. Second, we investigate the observability of the Higgs mode as the function of temperature in the unitary regime since the superfluid transition temperature in this region is reachable in the present experiments.

This paper is organized as follows. In Sec. II, we describe the theory that is employed to investigate the problem. In Sec. III, we calculate the spectral functions of the amplitude and phase fluctuations and discuss the spectral weight transfer of the Higgs and Goldstone modes. In Sec. IV, we calculate the spectral function of the amplitude fluctuation at finite temperature and demonstrate the observability of the Higgs mode in the unitary regime. Finally, we give a brief conclusion in Sec. V.

\section{PATH-INTEGRAL DESCRIPTION OF THE BEC-BCS CROSSOVER}

The partition function that describes a system of two species of fermions can be written as $\mathcal{Z}=\int D\left[\bar{\psi}_{\sigma}, \psi_{\sigma}\right] e^{-S\left[\psi_{\sigma}, \psi_{\sigma}\right]}$, with the action

$$
\begin{aligned}
S\left[\bar{\psi}_{\sigma}, \psi_{\sigma}\right]= & \int d \tau d^{3} \mathbf{x}\left\{\bar{\psi}_{\sigma}\left(\partial_{\tau}-\frac{\nabla^{2}}{2 m}-\mu\right)\right. \\
& \left.\times \psi_{\sigma}-g \bar{\psi}_{\uparrow} \bar{\psi}_{\downarrow} \psi_{\downarrow} \psi_{\uparrow}\right\},
\end{aligned}
$$


where $\psi_{\sigma}$ are the fermionic fields with spin index $\sigma=\uparrow, \downarrow$. The parameter $g$ is the contact interaction between fermions of opposite spins. It is related to the $s$-wave scattering length $a_{s}$ by $1 / g=-m / 4 \pi a_{s}+\sum_{\mathbf{k}} 1 / 2 \epsilon_{\mathbf{k}}$, with $\epsilon_{\mathbf{k}}=\mathbf{k}^{2} / 2 m$. Introducing an auxiliary field $\Delta(\tau, \mathbf{x})$, which represents the Cooper pair field, the four-fermion interaction term can be decoupled in the Cooper channel using the Hubbard-Stratonovich transformation. After integrating out the fermionic fields $\psi_{\sigma}$, one obtains an effective theory of the Cooper pair field $\Delta$ as

$$
\mathcal{Z}=\int D(\bar{\Delta}, \Delta) \exp \left[-\frac{1}{g} \int d \tau d \mathbf{x}|\Delta|^{2}+\ln \operatorname{det} \hat{G}^{-1}\right],
$$

where

$$
\hat{G}^{-1}=\left(\begin{array}{cc}
-\partial_{\tau}+\frac{\nabla^{2}}{2 m}+\mu & \Delta \\
\bar{\Delta} & -\partial_{\tau}-\frac{\nabla^{2}}{2 m}-\mu
\end{array}\right)
$$

is the Gor'kov Green's function.
To investigate the collective modes in the superfluid state we write the field $\Delta(\tau, \mathbf{x})$ as $[23,24]$

$$
\Delta(\tau, \mathbf{x})=\Delta_{0}+\delta \phi(\tau, \mathbf{x})
$$

where $\Delta_{0}$ is the mean field and $\delta \phi(\tau, \mathbf{x})$ represents the fluctuations. we next expand the action up to the second order of fluctuations. In the momentum space the action can be cast as

$$
S\left[\delta \phi^{*}, \delta \phi\right]=\frac{1}{2} \sum_{i \omega_{n}} \int \frac{d^{3} \mathbf{k}}{(2 \pi)^{3}} \bar{\Phi}\left(i \omega_{n}, \mathbf{k}\right) \mathbf{M}\left(i \omega_{n}, \mathbf{k}\right) \Phi\left(i \omega_{n}, \mathbf{k}\right),
$$

where we rearrange the fluctuation in the form of a spinor as $\bar{\Phi}\left(i \omega_{n}, \mathbf{k}\right)=\left[\delta \phi^{*}\left(i \omega_{n}, \mathbf{k}\right), \delta \phi\left(-i \omega_{n},-\mathbf{k}\right)\right]$ and $i \omega_{n}=i 2 n \pi / \beta$ is the bosonic Matsubara frequency. The inverse propagator $\mathbf{M}$ is given by

$$
\begin{aligned}
\mathbf{M}_{11}\left(i \omega_{n}, \mathbf{k}\right)= & \mathbf{M}_{22}\left(-i \omega_{n},-\mathbf{k}\right)=-\frac{m}{4 \pi a_{s}}+\int \frac{d^{3} \mathbf{q}}{(2 \pi)^{3}}\left\{\left[1-f-f^{\prime}\right]\left(\frac{u^{2} u^{\prime 2}}{i \omega_{n}-E-E^{\prime}}-\frac{v^{2} v^{\prime 2}}{i \omega_{n}+E+E^{\prime}}\right)\right. \\
& \left.+\left[f-f^{\prime}\right]\left(\frac{v^{2} u^{\prime 2}}{i \omega_{n}+E-E^{\prime}}-\frac{u^{2} v^{\prime 2}}{i \omega_{n}-E+E^{\prime}}\right)+\frac{1}{2 \epsilon_{\mathbf{k}}}\right\}, \\
\mathbf{M}_{12}\left(i \omega_{n}, \mathbf{k}\right)= & \mathbf{M}_{21}\left(i \omega_{n}, \mathbf{k}\right)=\int \frac{d^{3} \mathbf{q}}{(2 \pi)^{3}}\left\{\left[1-f-f^{\prime}\right]\left(\frac{u v u^{\prime} v^{\prime}}{i \omega_{n}+E+E^{\prime}}-\frac{u v u^{\prime} v^{\prime}}{i \omega_{n}-E-E^{\prime}}\right)\right. \\
& \left.+\left[f-f^{\prime}\right]\left(\frac{u v u^{\prime} v^{\prime}}{i \omega_{n}+E-E^{\prime}}-\frac{u v u^{\prime} v^{\prime}}{i \omega_{n}-E+E^{\prime}}\right)\right\} .
\end{aligned}
$$

In the above expressions we use the conventional notation $u=u_{\mathbf{q}}, v=v_{\mathbf{q}}, E=E_{\mathbf{q}}$ and $u^{\prime}=u_{\mathbf{q}+\mathbf{k}}, v^{\prime}=v_{\mathbf{q}+\mathbf{k}}, E^{\prime}=E_{\mathbf{q}+\mathbf{k}}$, where $E_{\mathbf{q}}=\sqrt{\xi_{\mathbf{q}}^{2}+\Delta_{0}^{2}}, v_{\mathbf{q}}^{2}=1-u_{\mathbf{q}}^{2}=\left(1-\xi_{\mathbf{q}} / E_{\mathbf{q}}\right) / 2$, and $\xi_{\mathbf{q}}=$ $\epsilon_{\mathbf{q}}-\mu . f=1 /[\exp (\beta E)+1]$ and $f^{\prime}=1 /\left[\exp \left(\beta E^{\prime}\right)+1\right]$ are the Fermi distributions.

\section{THE SPECTRAL WEIGHT FUNCTION OF THE HIGGS MODE AT TEMPERATURE $\boldsymbol{T}=0$}

To investigate the observability of the Higgs mode it is useful to separate the fluctuation $\delta \phi$ into real and imaginary parts $\delta \phi(\tau, \mathbf{x})=\left[\delta_{a}(\tau, \mathbf{x})+i \delta_{p}(\tau, \mathbf{x})\right] / \sqrt{2}$. Then $\delta_{a}$ and $\delta_{p}$ describe the amplitude and phase fluctuations, respectively. After a rotation the effective action can be written in the form

$$
S\left[\delta_{a}, \delta_{p}\right]=\frac{1}{2} \sum_{i \omega_{n}} \int \frac{d^{3} \mathbf{k}}{(2 \pi)^{3}}\left[\delta_{a}^{*}, \delta_{p}^{*}\right] \mathbf{Q}\left(i \omega_{n}, \mathbf{k}\right)\left[\begin{array}{l}
\delta_{a} \\
\delta_{p}
\end{array}\right],
$$

where the inverse propagator $\mathbf{Q}\left(i \omega_{n}, \mathbf{k}\right)$ is expressed in terms of $\mathbf{M}_{i j}\left(i \omega_{n}, \mathbf{k}\right)$ as

$$
\mathbf{Q}=\left[\begin{array}{cc}
\mathbf{M}_{\mathbf{1 1}}^{\mathbf{E}}+\mathbf{M}_{12} & i \mathbf{M}_{11}^{O} \\
-i \mathbf{M}_{11}^{O} & \mathbf{M}_{11}^{E}-\mathbf{M}_{12}
\end{array}\right]
$$

Here we redefine $\mathbf{M}_{11}^{E}=\mathbf{M}_{11}+\mathbf{M}_{22}$ and $\mathbf{M}_{11}^{O}=\mathbf{M}_{11}-\mathbf{M}_{22}$. The indices $\mathrm{E}$ and $\mathrm{O}$ denote even and odd in $i \omega_{n}$.
In this section we study the spectral weight transfer at zero temperature. At the $T=0$ limit the Fermi distribution functions $f$ and $f^{\prime}$ in Eq. (6) vanish. To study the dispersion relation and the spectral weight properties of the collective modes one usually takes the analytic continuation $i \omega_{n} \rightarrow$ $\omega+i 0^{+}$of the inverse propagator $\mathbf{Q}\left(\omega+i 0^{+}, \mathbf{k}\right)$ and then expands it in terms of small momentum $\mathbf{k}$ and energy $\omega$ [23] up to second order. However, in our calculation based on mean-field theory the expansion in small $\omega$ is not legitimate. While we expand $\mathbf{Q}\left(\omega+i 0^{+}, \mathbf{k}\right)$ in terms of small $\omega$, we actually treat $\omega / \min \left\{E_{\mathbf{k}}\right\}$ as a small parameter, where $\min \left\{E_{\mathbf{k}}\right\}$ is the minimum value of $E_{\mathbf{k}}$. The Higgs mode gap is of order $\Delta_{0}$. Hence, we are interested in the energy scale of $\omega \sim \Delta_{0}$. In this case $\omega / \min \left\{E_{\mathbf{k}}\right\}$ might not be a small parameter. For example, at the BCS limit $\min \left\{E_{\mathbf{k}}\right\}$ is of order $\Delta_{0}$ around the Fermi surface; then $\omega / \min \left\{E_{\mathbf{k}}\right\} \sim 1$. In our work we will directly calculate the spectral weight function from the inverse propagator $\mathbf{Q}\left(\omega+i 0^{+}, \mathbf{k}\right)$ without small $\omega$ and $\mathbf{k}$ expansion. After taking the analytic continuation $i \omega_{n} \rightarrow \omega+i 0^{+}$the matrix elements of $\mathbf{Q}(\omega, \mathbf{k})$ can be explicitly written as

$$
\begin{aligned}
\mathbf{Q}_{11}(\omega, \mathbf{k})= & -\frac{m}{4 \pi a_{s}}+\int \frac{d^{3} \mathbf{k}}{(2 \pi)^{3}} \\
& \times\left\{\frac{E+E^{\prime}}{2 E E^{\prime}} \frac{E E^{\prime}+\xi \xi^{\prime}-\Delta_{0}^{2}}{\left(\omega+i 0^{+}\right)^{2}-\left(E+E^{\prime}\right)^{2}}+\frac{1}{2 \epsilon_{\mathbf{k}}}\right\},
\end{aligned}
$$




$$
\begin{aligned}
\mathbf{Q}_{22}(\omega, \mathbf{k})= & -\frac{m}{4 \pi a_{s}}+\int \frac{d^{3} \mathbf{k}}{(2 \pi)^{3}} \\
& \times\left\{\frac{E+E^{\prime}}{2 E E^{\prime}} \frac{E E^{\prime}+\xi \xi^{\prime}+\Delta_{0}^{2}}{\left(\omega+i 0^{+}\right)^{2}-\left(E+E^{\prime}\right)^{2}}+\frac{1}{2 \epsilon_{\mathbf{k}}}\right\}, \\
\mathbf{Q}_{12}(\omega, \mathbf{k})= & \mathbf{Q}_{21}(-\omega,-\mathbf{k})=i \int \frac{d^{3} \mathbf{k}}{(2 \pi)^{3}} \\
& \times \frac{E \xi^{\prime}+E^{\prime} \xi}{4 E E^{\prime}} \frac{\omega}{\left(\omega+i 0^{+}\right)^{2}-\left(E+E^{\prime}\right)^{2}} .
\end{aligned}
$$

First, we will study the collective modes at the BCS limit. At zero temperature the off-diagonal term in Eq. (8) can be calculated as $\mathbf{Q}_{12}=i \mathbf{M}_{11}^{O}=i \int \frac{d^{3} \mathbf{k}}{(2 \pi)^{3}} \frac{E \xi^{\prime}+E^{\prime} \xi}{4 E E^{\prime}} \frac{\omega}{\omega^{2}-\left(E+E^{\prime}\right)^{2}}$. At the BCS limit the integrand in $\mathbf{Q}_{12}$ is approximately an odd function of $\mathbf{k}$ with respect to the axis $\epsilon_{\mathbf{k}}=\mu$. Then when one takes the integration of it, the positive and negative parts will cancel out each other. That is, the off-diagonal term $\mathbf{Q}_{12}$ vanishes. This manifests the particle-hole symmetry at the BCS limit. The spectrum of the collective modes can be calculated from the equation $\operatorname{det}[\mathbf{Q}(\omega, \mathbf{k})]=0$. As a result, $\mathbf{Q}_{11}$ and $\mathbf{Q}_{22}$ decouple. It is straightforward to see that we have two modes defined by $\operatorname{Re} \mathbf{Q}_{11}=0$ and $\operatorname{Re} \mathbf{Q}_{22}=0$. If we take the limit $\mathbf{q}=0$ and $\omega=2 \Delta_{0}$, the equation $\operatorname{Re} \mathbf{Q}_{11}=0$ reduces to the gap equation

$$
-\frac{m}{4 \pi a_{s}}=\int \frac{d^{3} \mathbf{k}}{(2 \pi)^{3}}\left\{\frac{1}{2 E}-\frac{1}{2 \epsilon_{\mathbf{k}}}\right\} .
$$

Using the gap equation, we can eliminate the term $m / 4 \pi a_{s}$ in $\operatorname{Re} \mathbf{Q}_{11}=0$ and finally obtain the equation $\int \frac{d^{3} \mathbf{k}}{(2 \pi)^{3}}\left\{\frac{E+E^{\prime}}{2 E E^{\prime}} \frac{E E^{\prime}+\xi \xi^{\prime}-\Delta_{0}^{2}}{\omega^{2}-\left(E+E^{\prime}\right)^{2}}+\frac{1}{2 E}\right\}$. In the limit of small $\mathbf{q}$, this equation can be rearranged as

$$
\left(\omega^{2}-\frac{1}{3} v_{F}^{2} q^{2}-4 \Delta_{0}^{2}\right) \int \frac{d^{3} \mathbf{k}}{(2 \pi)^{3}} \frac{1}{2 E\left(\omega^{2}-4 E^{2}\right)},
$$

where $v_{F}$ is the Fermi velocity. This equation explicitly shows that there is a collective mode with the dispersion relation, $\omega^{2}=\frac{1}{3} v_{F}^{2} q^{2}+4 \Delta_{0}^{2}$. This is the Higgs mode. The gap is $2 \Delta_{0}$. Analogously, we can obtain the dispersion relation of the gapless Goldstone mode as $\omega^{2}=\frac{1}{3} v_{F}^{2} q^{2}$ from the equation $\operatorname{Re} \mathbf{Q}_{22}=0$.

However, as one approaches the unitary regime, the offdiagonal term $\mathbf{Q}_{12}$ is not negligible anymore. A general discussion shows that the nonvanishing off-diagonal term is an indication of the breakdown of Lorentz invariance. Basically, after symmetry breaking, the phase and amplitude fluctuations decouple in the theory with Lorentz invariance. Hence, a phase and amplitude fluctuation coupled term like $\mathbf{Q}_{12}$ will break the Lorentz invariance. For a detailed discussion please refer to Refs. [1,21]. In Fig. 1 we compare $\operatorname{Im} \mathbf{Q}_{12}$ with the energy term $W \equiv \omega^{2} \int \frac{d^{3} \mathbf{k}}{(2 \pi)^{3}} \frac{1}{2 E\left(\omega^{2}-4 E^{2}\right)}$ in $\operatorname{Re} \mathbf{Q}_{11}$ and $\operatorname{Re} \mathbf{Q}_{22}$. It clearly shows that as one approaches the unitary regime from the BCS limit, the ratio $\left|\operatorname{Im} \mathbf{Q}_{12} / W\right|$ gradually increases from zero, which manifestly demonstrates the breakdown of the Lorentz invariance.

Hence, close to the unitary regime the phase and amplitude fluctuations couple together, and we cannot easily extract the collective modes as in Eq. (11). To investigate the behavior of the eigenmodes we evaluate the spectral weight function of $\delta_{a}$

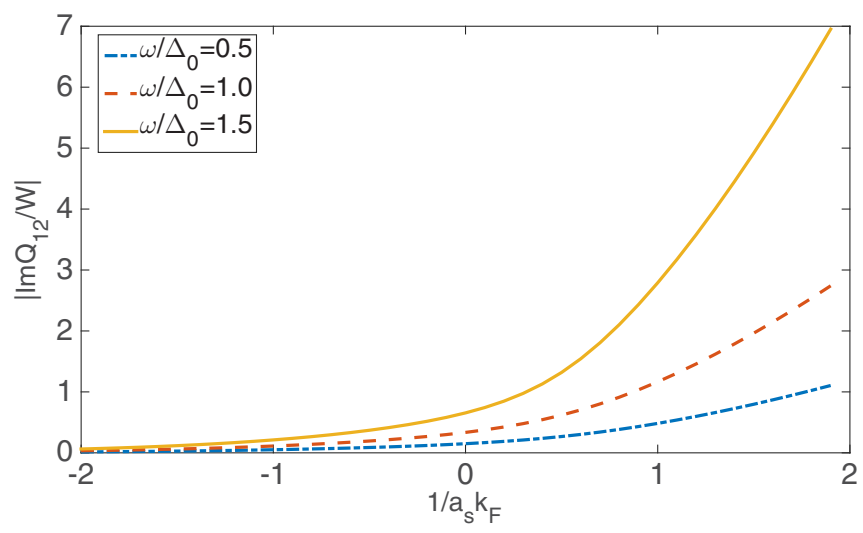

FIG. 1. The comparison of the off-diagonal term $\operatorname{Im} \mathbf{Q}_{12}$ and the energy term $W=\omega^{2} \int \frac{d^{3} \mathbf{k}}{(2 \pi)^{3}} \frac{1}{2 E\left(\omega^{2}-4 E^{2}\right)}$ in $\operatorname{Re} \mathbf{Q}_{11}$ and $\operatorname{Re} \mathbf{Q}_{22}$ for $\omega / \Delta_{0}=0.5,1$, and 1.5 .

and $\delta_{p}$, which can be calculated from the imaginary part of the propagators $\left\langle\delta_{a}^{*} \delta_{a}\right\rangle$ and $\left\langle\delta_{p}^{*} \delta_{p}\right\rangle$ after the analytical continuation.

$$
\begin{aligned}
& A_{a a}(\omega, \mathbf{k})=-\frac{1}{\pi} \operatorname{Im} \frac{\mathbf{Q}_{22}(\omega, \mathbf{k})}{\mathbf{Q}_{11}(\omega, \mathbf{k}) \mathbf{Q}_{22}(\omega, \mathbf{k})-\mathbf{Q}_{12}(\omega, \mathbf{k}) \mathbf{Q}_{21}(\omega, \mathbf{k})}, \\
& A_{p p}(\omega, \mathbf{k})=-\frac{1}{\pi} \operatorname{Im} \frac{\mathbf{Q}_{11}(\omega, \mathbf{k})}{\mathbf{Q}_{11}(\omega, \mathbf{k}) \mathbf{Q}_{22}(\omega, \mathbf{k})-\mathbf{Q}_{12}(\omega, \mathbf{k}) \mathbf{Q}_{21}(\omega, \mathbf{k})} .
\end{aligned}
$$

In Fig. 2 we demonstrate the spectral weight functions $A_{a a}(\omega, \mathbf{k})$ and $A_{p p}(\omega, \mathbf{k})$ for amplitude and phase fluctuations, respectively, at zero temperature. For simplicity we take the case of zero momentum. The four graphs are for different scattering lengths from the BCS to the Bose-Einstein condensate (BEC) side. We observe two features: (i) The spectral function of the phase fluctuation $A_{p p}(\omega, 0)$ only shows one sharp peak at $\omega=0$, which is the gapless Goldstone mode. At higher frequency $\omega>2 \Delta_{0}$ there is the two-particle continuum. As we have discussed, the phase and amplitude fluctuations

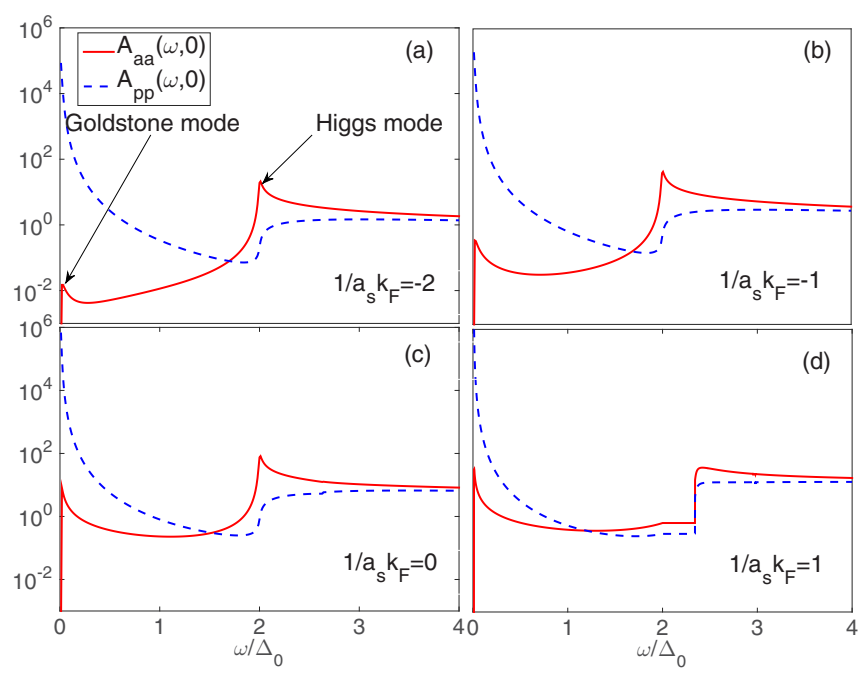

FIG. 2. The spectral weight functions $A_{a a}(\omega, 0)$ and $A_{p p}(\omega, 0)$ of amplitude and phase fluctuations at zero temperature. (a)-(d) Scattering lengths $1 / a_{s} k_{F}=-2,-1,0$, and 1 , respectively. 
strongly couple together in the unitary regime and BEC side. Then the phase fluctuation field is a superposition of two eigenmodes: the Goldstone and Higgs modes. However, the Higgs mode does not manifest itself in $A_{p p}(\omega, 0)$. This means the probe coupled to the phase fluctuation will not reveal any feature of the Higgs mode in the BEC-BCS crossover. (ii) The spectral function of the amplitude fluctuation $A_{a a}(\omega, 0)$ shows two peaks at the BCS limit and the unitary regime. One is the gapless Goldstone mode. The other one is the Higgs mode, which has a gap of $2 \Delta_{0}$ as calculated in Eq. (11). As one approaches the unitary regime from the BCS side, the spectral weight transfer from the Higgs to Goldstone mode is enhanced because the Lorentz invariance is lost, as we discussed in Ref. [21]. At the BEC limit the spectral weight of the Higgs mode is further diminished and finally overdamped by the two-particle continuum. However, around the unitary regime the spectral function $A_{p p}(\omega, 0)$ still possesses a feature of the gapped Higgs mode even though the Lorentz invariance is lost here.

\section{THE SPECTRAL WEIGHT FUNCTION AT FINITE TEMPERATURE}

In Fig. 2 we show that at zero temperature the spectral function $A_{a a}(\omega, 0)$ shows a clear peak of the Higgs mode at the unitary regime. In this section we want to investigate if the sharp-peak feature of the Higgs mode still exists at higher temperature around the unitary regime since in the present experiments the superfluid transition temperature can be reached in this region.

Assuming that the mean value of the Cooper pair field is $\Delta(T)$ at finite temperature, the effective action can be derived from Eq. (2) as $S_{\text {eff }}=\beta V \frac{\Delta(T)^{2}}{g}-\ln \operatorname{det} \hat{G}^{-1}$. Using the saddle-point condition $\partial S_{\text {eff }} / \partial \Delta(T)=0$, we obtain the gap equation as

$$
-\frac{m}{4 \pi a_{s}}=\int \frac{d^{3} \mathbf{k}}{(2 \pi)^{3}}\left\{\frac{1-2 f}{2 E}-\frac{1}{2 \epsilon_{\mathbf{k}}}\right\} .
$$

To solve the above equation for $\Delta(T)$ we need to determine the chemical potential $\mu(T)$. The total number of particles is $N=-k_{B} T \partial S_{\text {eff }} / \partial \mu(T)$. Then it is straightforward to calculate the number equation as

$$
n=\int \frac{d^{3} \mathbf{k}}{(2 \pi)^{3}}\left\{1-\frac{\xi}{E}(1-2 f)\right\} .
$$

Using Eqs. (13) and (14), the finite-temperature gap $\Delta(T)$ and chemical potential $\mu(T)$ can be solved self-consistently. At finite temperature the spectral functions $A_{a a}(\omega, \mathbf{k})$ and $A_{p p}(\omega, \mathbf{k})$ can still be calculated by Eq. (12), except that we use the finite-temperature matrix elements $Q_{i j}(\omega, \mathbf{k})$ and $M_{i j}(\omega, \mathbf{k})$ in Eqs. (6) and (8). The finite-temperature spectral function of the amplitude fluctuation $A_{a a}(\omega, \mathbf{k})$ at $1 / a_{s} k_{F}=-1$ is illustrated in Fig. 3. We investigate the case of $1 / a_{s} k_{F}=-1$ for two reasons. First, the superfluid transition temperature is reachable here in the realistic experiments. Second, our calculation of the critical temperature and $\Delta(T)$ is based on the mean-field theory. It is not accurate close to the unitarity and on the BEC side. However, at $1 / a_{s} k_{F}=-1$ the values are close to the Nozières-Schmitt-Rink calculation [25]. So

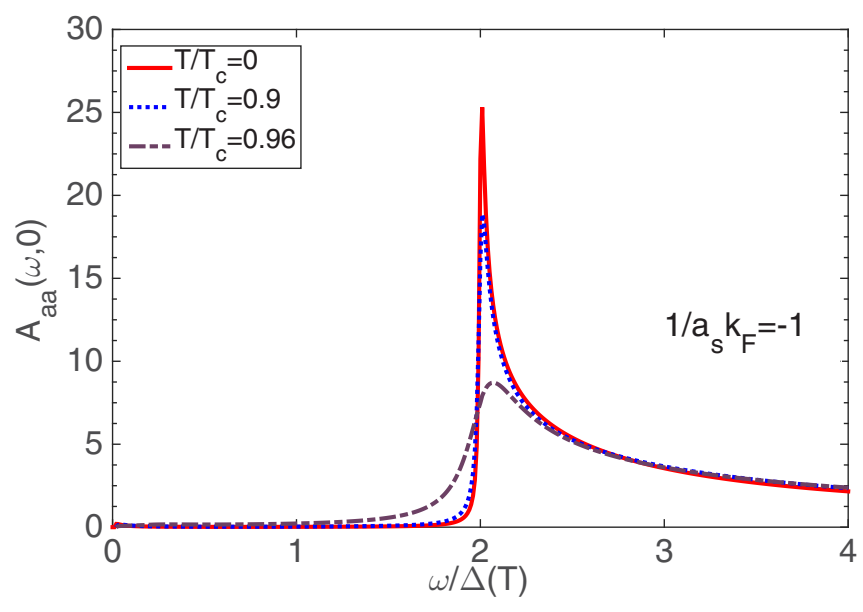

FIG. 3. The spectral weight function $A_{a a}(\omega, 0)$ at finite temperature. The scattering length is fixed at $1 / a_{s} k_{F}=-1$. As the temperature increases, the peak of the Higgs mode gets broadened. The superfluid transition temperature $T_{c}$ at $1 / a_{s} k_{F}=-1$ is $0.125 T_{F}$.

it is an appropriate point to study the finite-temperature spectral functions. We observe that as the temperature is increased, the peak of the Higgs mode is broadened due to the decay of the collective mode to the quasiparticles. At a temperature very close to $T_{c}$, for instance, $T / T_{c}=0.96$, the Higgs peak can get overdamped. However, for a wide range of temperature from $T=0$ to $T / T_{c}=0.9$ the Higgs mode still presents a nice feature of a sharp peak. This temperature range is reachable in the realistic experiment of a dilute Fermi gas. For example, in MIT's experiment [26] the temperature can be reduced to $T / T_{F} \simeq 0.05$ after evaporative cooling. The superfluid transition temperature at $1 / a_{s} k_{F}=-1$ is calculated as $T_{c}=0.125 T_{F}$ in our mean-field theory. This result demonstrates an opportunity to observe the Higgs mode in the fermionic superfluid with tunable interaction.

\section{CONCLUSIONS}

In summary, we have investigated the observability of the Higgs mode in the BCE-BCS crossover. The superfluid order parameter was treated as a spatially uniform mean field plus phase and amplitude fluctuations. We studied the observability of the Higgs mode by calculating the spectral weight function of the fluctuations. We emphasize that the usual analysis of low-frequency expansion [21,23] is not legitimate anymore in our mean-field study. In this work we directly calculated the spectral functions with the complete integral form of the matrix elements in Eq. (6). The zero-temperature study showed that the Higgs mode is totally overdamped by the two-particle continuum in the phase fluctuation spectral function $A_{p p}(\omega, \mathbf{k})$ for the whole BEC-BCS crossover. However, the signal of the Higgs mode was clear in the amplitude fluctuation spectral function $A_{p p}(\omega, \mathbf{k})$ even at the unitary regime. At finite temperature, we showed that in the temperature region that present experiments can reach the Higgs mode has a nice feature of a sharp peak around the unitary region. This suggests a potential observation of the Higgs mode in the system of dilute Fermi gases with tunable interactions. 


\section{ACKNOWLEDGMENTS}

We thank H. Zhai and S. Zhang for very useful discussions. The work is supported by "973" program (Grants No.
2010CB922904 and No. 2012CV821400), as well as National Science Foundation of China (Grants No. NSFC-1190024, No. 11175248 and No. 11104339), and the Strategic Priority Research Program of CAS (Grant No. XDB07000000).
[1] D. Pekker and C. M. Varma, Annu. Rev. Condens. Matter Phys. 6, 269 (2015).

[2] CMS Collaboration, Phys. Lett. B 716, 30 (2012).

[3] ATLAS Collaboration, Phys. Lett. B 716, 1 (2012).

[4] R. Sooryakumar and M. V. Klein, Phys. Rev. Lett. 45, 660 (1980).

[5] P. B. Littlewood and C. M. Varma, Phys. Rev. B 26, 4883 (1982).

[6] P. B. Littlewood and C. M. Varma, Phys. Rev. Lett. 47, 811 (1981).

[7] Ch. Rüegg, B. Normand, M. Matsumoto, A. Furrer, D. F. McMorrow, K. W. Kramer, H. U. Gudel, S. N. Gvasaliya, H. Mutka, and M. Boehm, Phys. Rev. Lett. 100, 205701 (2008).

[8] R. Matsunaga, Y. I. Hamada, K. Makise, Y. Uzawa, H. Terai, Z. Wang, and R. Shimano, Phys. Rev. Lett. 111, 057002 (2013).

[9] R. Matsunaga, N. Tsuji, H. Fujita, A. Sugioka, K. Makise, Y. Uzawa, H. Terai, Z. Wang, H. Aoki, and R. Shimano, Science 345, 1145 (2014).

[10] M. Endres, T. Fukuhara, D. Pekker, M. Cheneau, P. Schaub, C. Gross, E. Demler, S. Kuhr, and I. Bloch, Nature (London) 487, 454 (2012).

[11] C. M. Varma, J. Low Temp. Phys. 126, 901 (2002).

[12] Y. Barlas and C. M. Varma, Phys. Rev. B 87, 054503 (2013).

[13] S. D. Huber, B. Theiler, E. Altman, and G. Blatter, Phys. Rev. Lett. 100, 050404 (2008).
[14] L. Pollet and N. Prokof'ev, Phys. Rev. Lett. 109, 010401 (2012).

[15] D. Podolsky, A. Auerbach, and D. P. Arovas, Phys. Rev. B 84, 174522 (2011).

[16] D. Podolsky and S. Sachdev, Phys. Rev. B 86, 054508 (2012).

[17] K. Chen, L. Liu, Y. Deng, and L. Pollet, and N. Prokof'ev, Phys. Rev. Lett. 110, 170403 (2013).

[18] S. Gazit, D. Podolsky, and A. Auerbach, Phys. Rev. Lett. 110, 140401 (2013).

[19] A. Rançon and N. Dupuis, Phys. Rev. B 89, 180501(R) (2014).

[20] F. Rose, F. Léonard, and N. Dupuis, Phys. Rev. B 91, 224501 (2015).

[21] B. Liu, H. Zhai, and S. Zhang, Phys. Rev. A 93, 033641 (2016).

[22] T. Cea, C. Castellani, G. Seibold, and L. Benfatto, Phys. Rev. Lett. 115, 157002 (2015).

[23] J. R. Engelbrecht, M. Randeria, and C. A. R. Sá de Melo, Phys. Rev. B 55, 15153 (1997).

[24] R. B. Diener, R. Sensarma, and M. Randeria, Phys. Rev. A 77, 023626 (2008).

[25] P. Nozières and S. Schmitt-Rink, J. Low Temp. Phys. 59, 195 (1985).

[26] M. W. Zwierlein, J. R. Abo-Shaeer, A. Schirotzek, C. H. Schunck and W. Ketterle, Nature (London) 435, 1047 (2005). 